\title{
Weak Discrete Time Approximation of Stochastic Differential Equations with Time Delay
}

\author{
Uwe Küchler $^{1}$ and Eckhard Platen ${ }^{2}$
}

March 8, 2001

\begin{abstract}
The paper considers the derivation of weak discrete time approximations for solutions of stochastic differential equations with time delay. These are suitable for Monte Carlo simulation and allow the computation of expectations for functionals of stochastic delay equations. The suggested approximations converge in a weak sense.
\end{abstract}

Key words: Stochastic differential equations with time delay, discrete time approximation, weak convergence, simulation.

AMS Classification: $60 \mathrm{H} 10,65 \mathrm{C} 20$

\footnotetext{
${ }^{1}$ Humboldt-University Berlin, Institute of Mathematics, Unter den Linden 6, D - 10099 Berlin, Germany

${ }^{2}$ University of Technology Sydney, School of Finance \& Economics and School of Mathematical Sciences, PO Box 123, Broadway, NSW, 2007, Australia

This research was supported by the Deutsche Forschungsgemeinschaft (DFG), Sonderforschungsbereich 373, "Quantifikation und Simulation Ökonomischer Prozesse", HumboldtUniversität zu Berlin, the Australian Research Council and the University of Technology Sydney.
} 


\section{Introduction}

In many applications one has to take into consideration that often time will elapse between the cause of a certain phenomenon and its subsequent effect. Examples of this are seen in economics, finance, biology or medicine. Deterministic differential equations with time delay, also called differential delay equations (DDEs), are often used to model such phenomena. The theory and also some applications of DDEs are presented, for instance, in Kolmanovskii \& Myshkis (1992) or Hale \& Lunel (1993). Stochastic differential delay equations (SDDEs), are stochastic generalizations of DDEs. The basics of the theory for SDDEs can be found in Mohammed (1984, 1990), Mao (1994, 1997) and Mohammed \& Scheutzow (1990).

DDEs and therefore SDDEs have no simple explicit solutions or known distributions. In practice often functionals, that is expectations of functions of solutions of SDDEs, have to be computed. For ordinary stochastic differential equations (SDEs), Monte Carlo simulation methods have been developed as a powerful methodology to overcome the evaluation problem. Similarly, numerical procedures are also needed for the Monte Carlo simulation for SDDEs. First attempts in developing numerical methods for SDDEs have been made, for example, by Tudor \& Tudor (1987), Tudor (1989), Mao (1994), Baker \& Buckwar (1999) and Gilsing (2001). In Küchler \& Platen (2000), some explicit and implicit discrete time approximation methods for strong approximations of SDDEs were derived.

In this paper, we derive discrete time numerical methods for the weak approximation of SDDEs. This allows us to approximate moments, functionals and thus the distribution for the underlying process. A precise definition of weak convergence, including its order, will be given in Section 3. The resulting discrete time weak approximations permit the approximation of the expected value of a function of the solution of an SDDE by sampling from repeated simulations. Thus, weak schemes are tailored for Monte Carlo simulation. The efficiency of Monte Carlo methods strongly depends on the use of appropriate weak discrete time approximations. For a comparable order of convergence, weak schemes can be designed that are much simpler than their strong counterparts.

We consider a $d$-dimensional SDDE of the type

$$
d X(t)=a(X(t), X(t-r)) d t+\sum_{j=1}^{m} b^{j}(X(t), X(t-r)) d W^{j}(t)
$$

for $t \in[0, T], T<\infty$, which involves a fixed time delay $r$. The above SDDE has a structure that allows us to keep the resulting numerical methods relatively transparent. Equation (1.1) covers nonlinear, single delay, autonomous SDDEs with $m$ driving independent standard Wiener processes $W^{1}, \ldots, W^{m}$. Extensions of our results to nonautonomous coefficients and a finite number of different time delays are straightforward and therefore omitted.

Weak numerical methods for ordinary SDEs are well studied, see, for example, 
Kloeden \& Platen (1999) and Platen (1999) for a recent survey. In the present paper we will demonstrate that well-known weak approximation methods for SDEs can be extended to cover SDDE of the type described by (1.1).

The paper is organized as follows. In Section 2 we establish the existence and uniqueness of a solution of the SDDE (1.1). Section 3 introduces a weak convergence criterion. Sections 4 and 5 concentrate on weak discrete time approximations of solutions of SDDEs. These approximations include Euler schemes and weak second order schemes. Finally, in Section 6 we give a derivation of the presented schemes together with convergence theorems.

\section{Existence and Uniqueness of SDDEs}

At first let us establish the existence and uniqueness of the solution of the $d$ dimensional SDDE (1.1). Here we have a constant finite time delay $r>0$. The given $d$-dimensional initial segment

$$
\xi=\{\xi(s), s \in[-r, 0]\}
$$

is assumed to be right continuous having left hand limits. Let a filtered probability space $\left(\Omega, \mathcal{F}, \underline{\mathcal{F}}=\left(\mathcal{F}_{t}\right)_{t \in[0, T]}, P\right)$ be given that fulfills the usual conditions, see Protter (1990). The process $W=\{W(t), t \in[0, T]\}$ is supposed to be an $m$ -

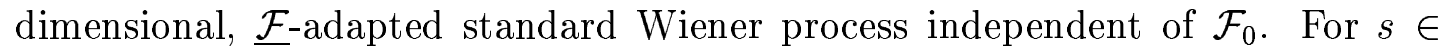
$[-r, 0]$ all values $\xi(s)$ of the initial segment are assumed to be $\mathcal{F}_{0}$-measurable.

The drift coefficient $a: \Re^{d} \times \Re^{d} \rightarrow \Re^{d}$ and the diffusion coefficient $b^{j}: \Re^{d} \times$ $\Re^{d} \rightarrow \Re^{d}, j \in\{1,2, \ldots, m\}$, are given $d$-dimensional sufficiently regular vector functions, as specified later on in the text.

The SDDE (1.1) can then be written in integrated form as

$$
X(t)=X(0)+\int_{0}^{t} a(X(s), X(s-r)) d s+\sum_{j=1}^{m} \int_{0}^{t} b^{j}(X(s), X(s-r)) d W^{j}(s)
$$

for $t \in[0, T]$. Here the initial segment $\xi$, see (2.1), enters the equation through the initial condition

$$
X(u)=\xi(u)
$$

for $u \in[-r, 0]$.

A $d$-dimensional process $X=\{X(t), t \in[-r, T]\}$ is called a solution of the SDDE (1.1) with initial segment $\xi$ if $X(t)$ is $\mathcal{F}_{t^{-}}$-measurable for all $t \in[0, T], X$ is continuous on $[0, T]$ and the equations (2.2) and (2.3) are satisfied. 
We denote by $|\cdot|$ the Euclidean norm. If any two solution processes $X^{(i)}=$ $\left\{X^{(i)}(t), t \in[-r, T]\right\}, i \in\{1,2\}$ with the same initial segment $\xi$ have, $P$-almost surely, the same path on $[0, T]$, that is

$$
P\left(\sup _{0 \leq t \leq T}\left|X^{(1)}(t)-X^{(2)}(t)\right|>0\right)=0,
$$

then we say that the solution (1.1) is unique for this initial segment $\xi$.

By $\mathbb{C}=\mathscr{C}\left([-r, 0], \Re^{d}\right)$ we denote the Banach space of all $d$-dimensional continuous functions $\eta$ on $[-r, 0]$ equipped with the supremums norm $\|\eta\|_{G}=\sup _{s \in[-r, 0]}$ $|\eta(s)|$. For every function $f \mid[-r, T] \rightarrow \Re^{d}$ and every $t \in[0, T]$ we define

$$
f_{t}=\left\{f_{t}(s):=f(t+s), s \in[-r, 0]\right\}
$$

as new function on $[-r, 0]$, the segment of $f$ at $t$. In this way, we obtain a segment valued function $t \rightarrow f_{t}$ for $t \in[0, T]$. Furthermore, we denote by $L_{2}\left(\Omega, \mathbb{C}, \mathcal{F}_{0}\right)$ the set of $\Re^{d}$-valued continuous processes $\eta=\{\eta(s), s \in[-r, 0]\}$ with $\eta(s)$ being $\mathcal{F}_{0}$-measurable for all $s \in[-r, 0]$ and

$$
E\left(\|\eta\|_{\mathscr{C}}^{2}\right)=E\left(\sup _{s \in[-r, 0]}|\eta(s)|^{2}\right)<\infty .
$$

To ensure the existence of a unique solution of (1.1) it is appropriate to formulate the following conditions.

$\left(L_{1}\right)$ (Lipschitz Condition): There exists a constant $K \in(0, \infty)$, such that

$$
\begin{aligned}
\left|a\left(x_{1}, y_{1}\right)-a\left(x_{2}, y_{2}\right)\right| & +\left|b^{1}\left(x_{1}, y_{1}\right)-b^{1}\left(x_{2}, y_{2}\right)\right| \\
+\left|b^{m}\left(x_{1}, y_{1}\right)-b^{m}\left(x_{2}, y_{2}\right)\right| & \leq K\left(\left|x_{2}-x_{1}\right|+\left|y_{2}-y_{1}\right|\right)
\end{aligned}
$$

for $t \in[0, T]$ and $x_{1}, x_{2}, y_{1}, y_{2} \in \Re^{d}$.

$\left(L_{2}\right)$ (Growth Condition): There exists a constant $K \in(0, \infty)$, such that

$$
|a(x, y)|^{2}+\left|b^{1}(x, y)\right|^{2}+\ldots+\left|b^{m}(x, y)\right|^{2} \leq K\left(1+|x|^{2}+|y|^{2}\right)
$$

for $x, y \in \Re^{d}$.

Proposition 2.1 Assume that the Lipschitz condition $\left(L_{1}\right)$ and the growth condition $\left(L_{2}\right)$ are satisfied and let $\xi$ be in $L_{2}\left(\Omega, \mathbb{C}, \mathcal{F}_{0}\right)$. Then the equation (1.1) with the initial segment $\xi$ has a unique solution $X=\{X(t), t \in[-r, T]\}$.

To ensure that all moments of $X(t)$ exist for all $t \in[0, T]$ it is sufficient to assume that

$$
E\left(\|\eta\|_{a}^{k}\right)<\infty
$$

for all $k \in\{2,3, \ldots\}$, which we suppose throughout the remainder of this paper. A proof of this result and the above proposition can be found in Mohammed (1984) and Mao (1997). 


\section{Order of Weak Convergence}

Let the end of our time horizon $T$ be greater than the delay time $r>0$. Suppose that $T=N_{0} r$ for some given integer $N_{0}$. The time step size $\Delta_{\ell}$ is given as

$$
\Delta_{\ell}=\frac{r}{\ell}
$$

where $\ell \in\{2,3, \ldots\}$. Throughout the paper, an equidistant time discretisation $(\tau)_{\Delta_{\ell}}=\left\{\tau_{n}: n \in\{-\ell,-\ell+1, \ldots, 0,1, \ldots, N\}\right\}$ of the time interval $[-r, T]$ is used with

$$
\tau_{n}=n \Delta_{\ell}
$$

and $N:=N_{0} \ell$.

For each given time $t \in[-r, T]$, let $n_{t}$ be the largest integer $n$ for which $\tau_{n}$ does not exceed $t$, that is $n_{t}=\max \left\{n \geq-\ell: \tau_{n} \leq t\right\}$. In particular, we have $n_{-r}=-\ell, n_{T}=N=N_{0} \ell$ and $\tau_{n_{T}}=T$.

Consider a process $Y^{\Delta_{\ell}}=\left\{Y^{\Delta_{\ell}}(t), t \in[-r, T]\right\}$, which is right continuous with left hand limits. We call $Y^{\Delta_{\ell}}$ a discrete time approximation with step size $\Delta_{\ell}$, provided it is based on a time discretization $(\tau)_{\Delta_{\ell}}$, and also the random variable $Y^{\Delta_{\ell}}\left(\tau_{n}\right)$ is $\mathcal{F}_{\tau_{n}}$-measurable for each $n \in\{1, \ldots, N\}$. Additionally, $Y^{\Delta_{\ell}}\left(\tau_{n+1}\right)$ must be expressed as a function of $Y^{\Delta_{\ell}}\left(\tau_{-\ell}\right), Y^{\Delta_{\ell}}\left(\tau_{-\ell+1}\right), \ldots, Y^{\Delta_{\ell}}\left(\tau_{n}\right)$, the discretization time $\tau_{n}$ and $\mathcal{F}_{\tau_{n+1}}$-measurable random variables $U_{n+1, j}, j \in\{1, \ldots, i\}$, where $i$ is finite. The discrete time approximations are not necessarily related to the Wiener processes $W$. However, defining $X$ and the discrete time approximations on the same filtered probability space does not restrict generality.

At given discretization times, we can calculate the values of the discrete time approximations recursively by using their approximate values obtained at earlier discretization times in conjunction with a finite number of independent random variables. In a Monte Carlo simulation for functionals of SDDEs, we use discrete time approximations evaluated only at discretization times. We are now interested in basic properties of such approximations. Since we only deal with the approximation of expectations of functions of solutions of SDDEs it is appropriate to introduce some kind of weak convergence. In this paper we shall use the concept of weak order convergence as defined in Section 9.7 in Kloeden \& Platen (1999). This allows us to classify given discrete time approximations.

We denote by $\mathcal{C}_{p}$ the set of all polynomials $g: \Re^{d} \rightarrow \Re$. A discrete time approximation $Y^{\Delta_{\ell}}$ converges weakly with order $\beta>0$ towards $X$ at time $T$ if for each $g \in \mathcal{C}_{p}$ there exists a constant $C$, which does not depend on $\Delta_{\ell}$, and some $L \in\{2,3, \ldots\}$ such that

$$
\left|E(g(X(T)))-E\left(g\left(Y^{\Delta_{\ell}}(T)\right)\right)\right| \leq C\left(\Delta_{\ell}\right)^{\beta}
$$

for each $\ell \geq L$. This criterion of weak order convergence relies on the polynomials as a set of test functions. Note that if a discrete time approximation converges weakly with some order $\beta$, then its moments also converge with this order. 


\section{Euler Schemes}

In this section we introduce some of the simplest discrete time weak approximations appropriate for the Monte Carlo simulation of functionals of solutions of SDDEs. We will also indicate the order of weak convergence of these schemes. The proof for these orders of convergence will be given in Section 6 . These schemes are generalizations of Euler schemes that are typically used for SDEs as treated in Section 14.1 in Kloeden \& Platen (1999). Besides the first method, outlined below, all other schemes are different from the strong schemes for SDDEs described in Küchler \& Platen (2000).

For the weak approximation of SDDEs, the Euler scheme is probably the most well-known method that one would consider. In the general multi-dimensional case $d, m \in\{1,2, \ldots\}$ the Euler scheme has the form

$$
Y_{n+1}=Y_{n}+a\left(Y_{n}, Y_{n-\ell}\right) \Delta+\sum_{j=1}^{m} b^{j}\left(Y_{n}, Y_{n-\ell}\right) \Delta W_{n}^{j}
$$

with Wiener process increment

$$
\Delta W_{n}^{j}=W^{j}\left(\tau_{n+1}\right)-W^{j}\left(\tau_{n}\right)
$$

for $n \in\{0,1, \ldots, N-1\}$ and $j \in\{1, \ldots, m\}$. Here we use the initial values

$$
Y_{i}=X\left(\tau_{i}\right)=\xi\left(\tau_{i}\right)
$$

for $i \in\{-\ell,-\ell+1, \ldots, 0\}$. For simplicity, here $Y_{n}=Y^{\Delta_{\ell}}\left(\tau_{n}\right)$, which is used throughout the following.

The one step increment of the Euler scheme (4.1), when specified for an SDE, corresponds to the truncated stochastic Taylor expansion, also called WagnerPlaten expansion, see Chapter 5 in Kloeden \& Platen (1999). It contains only the weighted time increment and weighted increments of the driving Wiener process components. From the convergence results in Section 6, the Euler scheme has order of weak convergence $\beta=1.0$ for sufficiently regular drift and diffusion coefficients. This means that the Euler scheme (4.1) is an order 1.0 weak scheme.

For weak convergence we need only to approximate the measure that is induced by the solution $X$ of the $\operatorname{SDDE}(2.2)$. As we will prove in Section 6, this allows us to replace the Gaussian increments $\Delta W_{n}^{j}$ in (4.1) by some simpler random variables $\Delta \hat{W}_{n}^{j}$ with appropriate moment properties to obtain the corresponding order of weak convergence. This leads us to the simplified Euler scheme for the $k$ th component of $Y$ in the form

$$
Y_{n+1}^{k}=Y_{n}^{k}+a^{k}\left(Y_{n}, Y_{n-\ell}\right) \Delta+\sum_{j=1}^{m} b^{k, j}\left(Y_{n}, Y_{n-\ell}\right) \Delta \hat{W}_{n}^{j}
$$


with initial values as described in (4.3). To ensure order $\beta=1.0$ weak convergence the random variables $\Delta \hat{W}_{n}^{j}$ for $j \in\{1,2, \ldots, m\}$ are here assumed to be independent $\mathcal{F}_{\tau_{n+1}}$-measurable random variables with moments satisfying the condition

$$
\left|E\left(\Delta \hat{W}_{n}^{j}\right)\right|+\left|E\left(\left(\hat{W}_{n}^{j}\right)^{3}\right)\right|+\left|E\left(\left(\hat{W}_{n}^{j}\right)^{2}\right)-\Delta\right| \leq K \Delta^{2}
$$

for some constant $K$. The simplest example of such a random variable $\Delta \hat{W}_{n}^{j}$ to be used in (4.4) is a two-point distributed random variable with

$$
P\left(\Delta \hat{W}_{n}^{j}= \pm \sqrt{\Delta}\right)=\frac{1}{2} .
$$

Obviously, the above two-point distributed random variables satisfy the moment conditions required by the simplified Euler scheme (4.4). With the choice (4.5), the simplified Euler scheme resembles a random walk.

\section{$5 \quad$ Second Order Explicit Weak Schemes}

In the case of SDEs, one obtains higher order weak schemes by including further multiple stochastic integrals from the Wagner-Platen expansion into the one step approximation, see Section 5.5 in Kloeden \& Platen (1999). Similarly, higher order weak schemes can be constructed for SDDEs. The objective is to include more information about the probability measure of the underlying delay process into the weak scheme to achieve a higher order of weak convergence. In this paper we consider approximations of order $\beta$ no greater than 2.0 to keep the schemes reasonably simple. It will become clear in what follows that higher order weak discrete time approximations can be similarly obtained. We assume that the drift and diffusion coefficients in (1.1) are sufficiently smooth such that the following schemes are well defined.

As a first one-dimensional example for a second order scheme we introduce the 
order 2.0 weak scheme in the case, $d=m=1$, which is defined by the relation

$$
\begin{aligned}
Y_{n+1}= & Y_{n}+a\left(Y_{n}, Y_{n-\ell}\right) \Delta+b\left(Y_{n}, Y_{n-\ell}\right) \Delta W_{n}^{1} \\
+ & b\left(Y_{n}, Y_{n-\ell}\right) \frac{\partial}{\partial Y_{n}} b\left(Y_{n}, Y_{n-\ell}\right) \frac{\left(\left(\Delta W_{n}^{1}\right)^{2}-\Delta\right)}{2} \\
+ & {\left[a\left(Y_{n}, Y_{n-\ell}\right) \frac{\partial a\left(Y_{n}, Y_{n-\ell}\right)}{\partial Y_{n}}+\frac{1}{2}\left(b\left(Y_{n}, Y_{n-\ell}\right)\right)^{2} \frac{\partial^{2} a\left(Y_{n}, Y_{n-\ell}\right)}{\left(\partial Y_{n}\right)^{2}}\right] \frac{\Delta^{2}}{2} } \\
+ & {\left[b\left(Y_{n}, Y_{n-\ell}\right) \frac{\partial a\left(Y_{n}, Y_{n-\ell}\right)}{\partial Y_{n}}\right.} \\
+ & \left.a\left(Y_{n}, Y_{n-\ell}\right) \frac{\partial b\left(Y_{n}, Y_{n-\ell}\right)}{\partial Y_{n}}+\frac{1}{2}\left(b\left(Y_{n}, Y_{n-\ell}\right)\right)^{2} \frac{\partial^{2} b\left(Y_{n}, Y_{n-\ell}\right)}{\left(\partial Y_{n}\right)^{2}}\right] \frac{\Delta}{2} \Delta W_{n}^{1} \\
+ & \mathbf{1}_{\{n>\ell\}}\left(b \left(Y_{n-\ell}, Y_{n-2 \ell} \frac{\partial}{\partial Y_{n-\ell}} b\left(Y_{n}, Y_{n-\ell}\right) \frac{\left(\Delta W_{n}^{1} \Delta W_{n-\ell}^{1}-\Delta\right)}{2}\right.\right. \\
+ & {\left[a\left(Y_{n-\ell}, Y_{n-2 \ell}\right) \frac{\partial a\left(Y_{n}, Y_{n-\ell}\right)}{\partial Y_{n-\ell}}+\frac{1}{2}\left(b\left(Y_{n-\ell}, Y_{n-2 \ell}\right)\right)^{2} \frac{\partial^{2} a\left(Y_{n}, Y_{n-\ell}\right)}{\left(\partial Y_{n-\ell}\right)^{2}}\right] \frac{\Delta^{2}}{2} } \\
+ & {\left[b\left(Y_{n-\ell}, Y_{n-2 \ell}\right) \frac{\partial a\left(Y_{n}, Y_{n-\ell}\right)}{\partial Y_{n-\ell}}+a\left(Y_{n-\ell}, Y_{n-2 \ell}\right) \frac{\partial b\left(Y_{n}, Y_{n-\ell}\right)}{\partial Y_{n-\ell}}\right.} \\
& +\frac{1}{2}\left(b\left(Y_{n-\ell}, Y_{n-2 \ell))^{2}} \frac{\partial^{2} b\left(Y_{n}, Y_{n-\ell}\right)}{\left(\partial Y_{n-\ell}\right)^{2}} \frac{\Delta}{2} \Delta W_{n-\ell}^{1}\right)\right.
\end{aligned}
$$

Here $\mathbf{1}_{\{\cdot\}}$ is the indicator function and we use the random variables $\Delta W_{k}^{1}$ as defined in (4.2) for $k \in\{0,1, \ldots, N-1\}$. The important feature of the above second order weak scheme is that it involves increments of the Wiener process at the actual time $t$ as well as those from the delayed time $t-r$. Thus one has to store the previously generated random variables from the actual time until the delayed time.

To present a more general second order weak scheme we formally introduce the operators

$$
\begin{aligned}
L_{z}^{0}= & \sum_{\gamma=1}^{d} a^{\gamma}\left(Y_{n-z \ell}, Y_{n-(z+1) \ell}\right) \frac{\partial}{\partial Y_{n-z \ell}} \\
& +\frac{1}{2} \sum_{\gamma, \lambda=1}^{d} \sum_{j=1}^{m} b^{\gamma, j}\left(Y_{n-z \ell}, Y_{n-(z+1) \ell}\right) b^{\lambda, j}\left(Y_{n-z \ell}, Y_{n-(z+1) \ell}\right) \frac{\partial^{2}}{\partial Y_{n-z \ell}^{\gamma} \partial Y_{n-z \ell}^{\lambda}}
\end{aligned}
$$


and

$$
L_{z}^{j}=\sum_{\gamma=1}^{d} b^{\gamma, j}\left(Y_{n-z \ell}, Y_{n-(z+1) \ell}\right) \frac{\partial}{\partial Y_{n-z \ell}^{\gamma}}
$$

for $z \in\{0,1\}$ and $j \in\{1, \ldots, m\}$.

For the general multi-dimensional case with $d, m \in\{1,2, \ldots\}$, the $i$ th component of the order 2.0 weak scheme has the form

$$
\begin{aligned}
Y_{n+1}^{i}= & Y_{n}^{i}+a^{i}\left(Y_{n}, Y_{n-\ell}\right) \Delta+\sum_{j=1}^{m} b^{i, j}\left(Y_{n}, Y_{n-\ell}\right) \Delta \tilde{W}_{n}^{j} \\
& +\left[L_{0}^{0}+L_{1}^{0}\right] a^{i}\left(Y_{n}, Y_{n-\ell}\right) \frac{\Delta^{2}}{2} \\
& +\sum_{j=1}^{m}\left[L_{0}^{j} a^{i}\left(Y_{n}, Y_{n-\ell}\right)+\left(L_{0}^{0}+L_{1}^{0}\right) b^{i, j}\left(Y_{n}, Y_{n-\ell}\right)\right] \frac{\Delta}{2} \Delta \tilde{W}_{n}^{j} \\
& +\sum_{j=1}^{m} L_{1}^{j} a^{i}\left(Y_{n}, Y_{n-\ell}\right) \frac{\Delta}{2} \Delta \tilde{W}_{n-\ell}^{j} \\
& +\sum_{j_{1}, j_{2}=1}^{m}\left[L_{0}^{j_{1}} b^{i, j_{2}}\left(Y_{n}, Y_{n-\ell}\right) \frac{\left(\Delta \tilde{W}_{n}^{j_{1}} \Delta \tilde{W}_{n}^{j_{2}}+V_{n}^{j_{1}, j_{2}}\right)}{2}\right] \\
& \left.+L_{1}^{j_{1}} b^{i, j_{2}}\left(Y_{n}, Y_{n-\ell}\right) \frac{\left(\Delta \tilde{W}_{n-\ell}^{j_{1}} \Delta \tilde{W}_{n}^{j_{2}}-\Delta\right)}{2}\right]
\end{aligned}
$$

for $n \in\{0,1, \ldots, N-1\}, i \in\{1,2, \ldots, d\}$. Here $\Delta \tilde{W}_{k}^{j}$ for $k \in\{0, \ldots, N-1\}$ and $j \in\{1, \ldots, m\}$ are independent random variables with

$$
\begin{aligned}
& \left|E\left(\Delta \tilde{W}_{k}^{j}\right)\right|+\left|E\left(\left(\Delta \tilde{W}_{k}^{j}\right)^{3}\right)\right|+\left|E\left(\left(\Delta \tilde{W}_{k}^{j}\right)^{5}\right)\right| \\
& +\left|E\left(\left(\Delta \tilde{W}_{k}^{j}\right)^{2}\right)-\Delta\right|+\left|E\left(\left(\Delta \tilde{W}_{k}^{j}\right)^{4}\right)-3 \Delta^{2}\right| \leq K \Delta^{3}
\end{aligned}
$$

for some constant $K<\infty$. Obviously, a Gaussian random variable with mean zero and variance $\Delta$ satisfies condition (5.5). Other simple random variables that satisfy condition (5.5) are three point distributed random variables $\Delta \tilde{W}_{k}^{j}$ with

$$
\begin{aligned}
P\left(\Delta \tilde{W}_{k}^{j}= \pm \sqrt{3 \Delta}\right) & =\frac{1}{6} \\
P\left(\Delta \tilde{W}_{k}^{j}=0\right) & =\frac{2}{3} .
\end{aligned}
$$


In (5.4), the quantities $V_{k}^{j_{1}, j_{2}}$ for $j_{1}, j_{2} \in\{1, \ldots, m\}$ and $k \in\{0, \ldots, N-1\}$, are assumed to be independent two-point distributed random variables with

$$
P\left(V_{k}^{j_{1}, j_{2}}= \pm \Delta\right)=\frac{1}{2}
$$

for $j_{2} \in\left\{1, \ldots, j_{1}-1\right\}$,

$$
V_{k}^{j_{1}, j_{1}}=-\Delta
$$

and

$$
V_{k}^{j_{1}, j_{2}}=-V_{k}^{j_{2}, j_{1}}
$$

for $j_{2} \in\left\{j_{1}+1, \ldots, m\right\}, j_{1} \in\{1, \ldots, m\}$ and $k \in\{0, \ldots, N-1\}$.

The scheme (5.4) covers also the one-dimensional scheme (5.1) and in the following section it will be shown to converge weakly with order $\beta=2.0$.

\section{Derivation of the Schemes}

For the schemes described above, the proof of the order of weak convergence is feasible since the solution of SDDE (1.1) can be expressed as a sequence of solutions of systems of SDEs without time delay. For this purpose we introduce the notation

$$
Z^{i, k}(t)=X(t-i r)
$$

for all $t \in[(k-1) r, k r], i \in\{0,1, \ldots, k\}$ and $k \in\left\{0,1, \ldots, N_{0}\right\}$ with $N_{0}=\frac{T}{r}$, where $X$ is the solution of (1.1). Then we have

$$
Z^{i, k}(k r)=Z^{i, k+1}(k r)
$$

for $k \in\left\{0,1, \ldots, N_{0}-1\right\}$ and $i \in\{0,1, \ldots, k\}$.

To simplify our notation in the following, we set

$$
b^{0}(x, y)=a(x, y)
$$

for all $x, y \in \Re^{d}$. Furthermore, we write

$$
W^{0}(t)=t
$$

for all $t \in[0, T]$, where $W^{0}=\left\{W^{0}(t), t \in[0, T]\right\}$ can be interpreted as the time process. 
From (1.1) we can recursively derive the following system of SDEs, with the notation of (6.1) and (6.2), where for $k \in\left\{0,1, \ldots, N_{0}\right\}$ and $t \in[(k-1) r, k r]$, we obtain

$$
\begin{aligned}
d Z^{0, k}(t) & =\sum_{j=0}^{m} b^{j}\left(Z^{0, k}(t), Z^{1, k}(t)\right) d W^{j}(t) \\
\vdots & \\
d Z^{k-1, k}(t) & =\sum_{j=0}^{m} b^{j}\left(Z^{k-1, k}(t), Z^{k, k}(t)\right) d W^{j}(t-(k-1) r)
\end{aligned}
$$

with

$$
\begin{gathered}
Z^{k, k}(t)=\xi(t-k r), \\
Z^{k-1, k-1}((k-1) r)=Z^{k-1, k}((k-1) r), \ldots, Z^{0, k-1}((k-1) r)=Z^{0, k}((k-1) r) .
\end{gathered}
$$

By construction we have now the representation

$$
X(t)=Z^{0, k}(t)
$$

for $t \in[(k-1) r, k r]$.

Thus we succeeded in (6.6) to express, for specified subintervals, the solution of the SDDE (1.1) as a solution of a multi-dimensional stochastic differential equation without time delay. This allows us to study the problem of the construction of weak discrete time approximations for the SDDE (1.1) as a problem for the corresponding system of SDEs (6.5), which must be considered on a finite number of time intervals. However, these time intervals are not changed by a refinement of our time discretization.

Let us now take an increment of the solution of the system of SDEs (6.5) for some discretization times $\tau_{n}, \tau_{n+1} \in[(k-1) r, k r], k \in\left\{0,1, \ldots, N_{0}\right\}$, that is

$$
\begin{aligned}
& Z^{0, k}\left(\tau_{n+1}\right)-Z^{0, k}\left(\tau_{n}\right)=\sum_{j=0}^{m} \int_{\tau_{n}}^{\tau_{n+1}} b^{j}\left(Z^{0, k}(t), Z^{1, k}(t)\right) d W^{j}(t) \\
& \vdots \\
& Z^{k-1, k}\left(\tau_{n+1}\right)-Z^{k-1, k}\left(\tau_{n}\right)=\sum_{j=0}^{m} \int_{\tau_{n}}^{\tau_{n+1}} b^{j}\left(Z^{k-1, k}(t), Z^{k, k}(t)\right) \\
& \cdot d W^{j}(t-(k-1) r),
\end{aligned}
$$

where $Z^{k, k}(t)=\xi(t-k r)$.

The Itô formula can be applied to the integrands of the components on the right hand side of the above equation. To do that efficiently we apply the operators 
$L_{z}^{p}$ introduced in (5.2) in the form

$$
\begin{aligned}
& L_{z}^{0}=\sum_{\gamma=1}^{d} a^{\gamma}\left(Z^{z, k}(u), Z^{z+1, k}(u)\right) \frac{\partial}{\partial Z^{z, k, \gamma}} \\
&+\frac{1}{2} \sum_{\gamma, \lambda=1}^{d} \sum_{j=1}^{m} b^{\gamma, j}\left(Z^{z, k}(u), Z^{z+1, k}(u)\right) \\
& \cdot b^{\lambda, j}\left(Z^{z, k}(u), Z^{z+1, k}(u)\right) \frac{\partial^{2}}{\partial Z^{z, k, \gamma} \partial Z^{z, k, \lambda}}
\end{aligned}
$$

and

$$
L_{z}^{j}=\sum_{\gamma=1}^{d} b^{\gamma, j}\left(Z^{z, k}(u), Z^{z+1, k}(u)\right) \frac{\partial}{\partial Z^{z, k, \gamma}}
$$

for $j \in\{1, \ldots, m\}$ and $z \in\{0,1, \ldots\}$. Taking into account that increments of Wiener processes on disjoint intervals are independent, we obtain for $j \in$ $\{0, \ldots, m\}, t \in[(k-1) r, k r]$ and $z \in\{0,1, \ldots, k-1\}$ for the $j$ th coefficients the representation

$$
\begin{aligned}
& b^{j}\left(t-z r, Z^{z, k}(t), Z^{z+1, k}(t)\right) \\
&= b^{j}\left(Z^{z, k}\left(\tau_{n}\right), Z^{z+1, k}\left(\tau_{n}\right)\right) \\
&+\sum_{p=0}^{m}\left(\int_{\tau_{n}}^{t} L_{z}^{p} b^{j}\left(Z^{z, k}(u), Z^{z+1, k}(u)\right) d W^{p}(u-z r)\right. \\
&\left.+\int_{\tau_{n}}^{t} L_{z+1}^{p} b^{j}\left(Z_{u}^{z, k}, Z_{u}^{z+1, k}\right) d W^{p}(u-(z+1) r)\right) .
\end{aligned}
$$

Let us now introduce the following notation for a double Itô integral of the form

$$
I_{(j, p), \tau, \varrho, z}=\int_{\tau}^{\varrho} \int_{\tau}^{s} d W^{j}(u-z) d W^{p}(s)
$$

for $0 \leq \tau<\varrho \leq T, z \in\{0, r\}$ and $j, p \in\{0, \ldots, m\}$. Here we interpret again the process $W^{0}$ as time and consequently cover in (6.11), all mixtures of time and Itô-Wiener integrals.

In (6.10), we apply the Itô formula again to $L_{z}^{p} b^{j}$ and $L_{z+1}^{p} b^{j}$. With (6.10), we 
then obtain from (6.7), the representation

$$
\begin{aligned}
& Z^{0, k}\left(\tau_{n+1}\right)-Z^{0, k}\left(\tau_{n}\right)=\sum_{j=0}^{m} b^{j}\left(Z^{0, k}\left(\tau_{n}\right), Z^{1, k}\left(\tau_{n}\right)\right)\left(W^{j}\left(\tau_{n+1}\right)-W^{j}\left(\tau_{n}\right)\right) \\
& +\sum_{j, p=0}^{m}\left\{L_{0}^{p} b^{j}\left(Z^{0, k}\left(\tau_{n}\right), Z^{1, k}\left(\tau_{n}\right)\right) I_{(p, j), \tau_{n}, \tau_{n+1}, 0}\right. \\
& \left.+L_{1}^{p} b^{j}\left(Z^{0, k}\left(\tau_{n}\right), Z^{1, k}\left(\tau_{n}\right)\right) I_{(p, j), \tau_{n}, \tau_{n+1}, r}\right\} \\
& +R^{0, k}\left(\tau_{n}\right) \\
& Z^{k-1, k}\left(\tau_{n+1}\right)-Z^{k-1, k}\left(\tau_{n}\right)=\sum_{j=0}^{m} b^{j}\left(Z^{k-1, k}\left(\tau_{n}\right), Z^{k, k}\left(\tau_{n}\right)\right) \\
& \cdot\left(W^{j}\left(\tau_{n+1}-(k-1) r\right)-W^{j}\left(\tau_{n}-(k-1) r\right)\right) \\
& +\sum_{j, p=0}^{m}\left\{L_{k-1}^{p} b^{j}\left(Z^{k-1, k}\left(\tau_{n}\right), Z^{k, k}\left(\tau_{n}\right)\right)\right. \\
& \cdot I_{(p, j), \tau_{n}-(k-1) r, \tau_{n+1}-(k-1) r, 0} \\
& +L_{k}^{p} b^{j}\left(Z^{k-1, k}\left(\tau_{n}\right), Z^{k, k}\left(\tau_{n}\right)\right) \\
& \left.\cdot I_{(p, j), \tau_{n}-k r, \tau_{n+1}-(k-1) r, r}\right\} \\
& +R^{k-1, k}\left(\tau_{n}\right) \text {. }
\end{aligned}
$$

Here the terms $R^{i, k}\left(\tau_{n}\right), i \in\{0,1, \ldots, k-1\}$, represent the remaining higher order terms that are not needed for a weak order $\beta=2.0$ discrete time approximation. In the above expansion (6.12), we now neglect these remaining higher order terms and obtain the increments of the order 2.0 weak Taylor approximation, see Section 14.2 in Kloeden \& Platen (1999), for the increments (6.7) of the SDE (6.5). For these order 2.0 weak Taylor approximations for SDEs there exist convergence theorems that we can apply, see Section 14.5 in Kloeden \& Platen (1999). Theorem 6.1 below will specify the conditions under which the weak order $\beta=2.0$ convergence is obtained.

It seems cumbersome to deal in a scheme with the full system of SDEs (6.5) and the resulting system of weak approximations. Fortunately, this is not necessary. Due to the structure of the expansion (6.12), one notes by the relations (6.1), (6.8) and (6.9), that the first component of the resulting scheme for the system of SDEs corresponds to the weak order 2.0 scheme (5.4) of SDDE (1.1). However, by construction, the other components in (6.12) repeat only the computation of approximate solutions for the delay equation (1.1) that were obtained in earlier 
subintervals. This means that these components are redundant and need not be recalculated. Therefore, the schemes (5.1) and (5.4) are derived.

Using the above derivation of the schemes (5.1) and (5.4) the following theorem follows from Theorem 14.5.1 and Theorem 14.5.2 in Kloeden \& Platen (1999).

For $\ell \in\{2,3, \ldots\}$, let us denote by $\bar{C}_{p}^{\ell}$ the set of $\ell$ times continuously differentiable functions $g: \Re^{d} \rightarrow \Re$, where $g$ and all of its partial derivatives of orders up to and including $\ell$ have polynomial growth.

Theorem 6.1 Suppose that the drift and diffusion coefficient functions satisfy the Lipschitz condition $L_{1}$ and the growth condition $L_{2}$. Furthermore, assume that $a^{i}$ and $b^{i, j}$ are from $\bar{C}_{p}^{6}$ for all $i \in\{1, \ldots, d\}$ and $j \in\{0, \ldots, m\}$ and that

$$
\left|L_{0}^{j_{1}} b^{i, j_{2}}(x, y)\right|+\left|L_{1}^{j_{1}} b^{i, j_{2}}(x, y)\right| \leq K(1+|x|+|y|)
$$

for $x, y \in \Re^{d}$ and $i \in\{1, \ldots, d\}, j_{1}, j_{2} \in\{0, \ldots, m\}$, here $K<\infty$. Then for each $g \in \bar{C}_{p}^{6}$ there exists for the schemes (5.1) and (5.4) a constant $K_{g}$, which does not depend on $\Delta$, such that

$$
\left|E(g(X(T)))-E\left(g\left(Y^{\Delta_{\ell}}(T)\right)\right)\right| \leq K_{g} \Delta^{2} .
$$

This means that these schemes converge weakly with order $\beta=2.0$.

If we neglect in (6.12), not only the remaining higher order terms as well as all the terms that refer to double integrals, then we obtain in the same manner as above a derivation of the Euler approximation (4.1) and the simplified Euler scheme (4.4). Again Theorem 14.5.1 and Theorem 14.5.2 in Kloeden \& Platen (1999) can be applied to prove the following result.

Theorem 6.2 Assume that the drift and diffusion coefficient functions satisfy the Lipschitz condition $L_{1}$ and the growth condition $L_{2}$ and are from $\bar{C}_{p}^{4}$. Then there exists for the Euler scheme (4.1) and the simplified Euler scheme (4.4) for each $g \in \bar{C}_{p}^{4}$ a constant $K_{g}$ not depending on $\Delta$, such that

$$
\left|E(g(X(T)))-E\left(g\left(Y^{\Delta_{\ell}}(T)\right)\right)\right| \leq K_{g} \Delta .
$$

The implementation of the weak methods outlined above is standard. In the case of second weak order schemes, one has to be able to recall the random numbers backwards for one delay period. Otherwise, the Monte Carlo simulation is very common and can be performed as described in Chapter 16 in Kloeden \& Platen (1999).

Using the methodology described, similar results can be obtained for other higher order weak schemes for SDDEs based on results for discrete time weak approximations for SDEs. In principle, this also applies to implicit schemes, which will be treated separately. 


\section{References}

Baker, C. T. H. \& E. Buckwar (1999). Introduction to the numerical analysis of stochastic delay differential equations. Technical report, University of Manchester, Dept. of Mathematics. Numerical Analysis Report 345.

Gilsing, H. (2001). On the stability of the Euler scheme for an affine stochastic differential equation with time delay. Technical report, Humboldt University, Berlin. (in German) Diskussion Paper No. 20, SFB 373.

Hale, J. K. \& V. S. M. Lunel (1993). Introduction to Functional-Differential Equations. Springer.

Kloeden, P. E. \& E. Platen (1999). Numerical Solution of Stochastic Differential Equations, Volume 23 of Appl. Math. Springer. Third corrected printing.

Kolmanovskii, V. \& A. Myshkis (1992). Applied Theory of Fundamental Differential Equations. Kluwer.

Küchler, U. \& E. Platen (2000). Strong discrete time approximation of stochastic differential equations with time delay. Math. Comput. Simulation 54, 189-205.

Mao, X. (1994). Exponential Stability of Stochastic Differential Equations. Marcel Dekker.

Mao, X. (1997). Stochastic Differential Equations and Applications. Horwood.

Mohammed, S.-E. A. (1984). Stochastic functional differential equations, Volume 99 of Research Notes in Mathematics. Pitman.

Mohammed, S.-E. A. (1990). Lyapunov exponents and stochastic flows of linear and affine hereditary systems. In Diffusion processes and related problems in analysis, Vol. II, Volume 27 of Progr. Probab., pp. 141-169. Birkhäuser.

Mohammed, S.-E. A. \& M. L. R. Scheutzow (1990). Lyapunov exponents and stationary solutions for affine stochastic delay equations. Stochastics Stochastics Rep. 29(2), 259-283.

Platen, E. (1999). An introduction to numerical methods for stochastic differential equations. Acta Numer. 8, 197-246.

Protter, P. (1990). Stochastic Integration and Differential Equations. Springer.

Tudor, C. (1989). Approximation of delay stochastic equations with constant retardation by usual Ito equations. Rev. Roumaine Math. Pures Appl. 34(1), 55-64.

Tudor, C. \& M. Tudor (1987). On approximation of solutions for stochastic delay equations. Stud. Cerc. Mat. 39(3), 265-274. 